\title{
COMMENTARY
}

\section{CXCR3 blockade: a novel anti-sepsis approach?}

\author{
Matthew J Delano and Lyle L Moldawer* \\ See related research by Herzig et al., http://ccforum.com/content/16/5/R168
}

\begin{abstract}
Blockade of the CXC chemokine receptor 3 (CXCR3) attenuates inflammation and improves survival in a murine model of near lethal polymicrobial sepsis. However, given the multitude of cellular responses and inflammatory mediators that orchestrate sepsis syndrome, more detailed investigations will be required before a complete understanding of the mechanism(s) of CXCR3 blockade and its therapeutic potential are revealed.
\end{abstract}

In the previous issue of Critical Care, Herzig and colleagues [1] set out to determine whether blockade of the CXC chemokine receptor 3 (CXCR3) attenuates inflammation and improves survival in a murine model of near lethal polymicrobial sepsis. Their results show that concomitant CXCR3 blockade and antibiotic therapy significantly improves survival when administered prior to and even shortly after sepsis. This study is a natural extension of the authors' prior work that demonstrates that CXCR3 blockade impedes lymphocyte trafficking, decreases systemic cytokine production and improves sepsis survival [2]. From this body of work, the authors conclude that CXCR3 inhibition should be considered a potential target for anti-sepsis therapies.

Although the authors' results are compelling, the conclusion that CXCR3 signaling is detrimental in polymicrobial sepsis must be accepted with caution. Others have previously investigated the impact of CXCR3 and its ligand, CXCL10, with strikingly dissimilar results, depending upon the model employed. In contrast to Herzig and colleagues' report, several prior investigations have found that CXCR3 and CXCL10 were necessary for survival in adult and neonatal murine polymicrobial sepsis models. Kunkle, Standiford and colleagues $[3,4]$ in separate reports demonstrated that CXCL10 blockade

*Correspondence: moldawer@surgery.ufl.edu

Department of Surgery, University of Florida, College of Medicine, Gainesville, Florida 32610, USA worsens survival in sepsis and pneumonia. Cuenca and colleagues [5] showed that CXCL10 concentrations increase in the peritoneum and blood of septic neonatal mice, that CXCL10 blockade and CXCR3 blockade worsen survival, and that adjuvant augmentation of CXCL10 is protective from sepsis mortality. In two consecutive reports, Kelly-Scumpia and colleagues from our laboratory implicated type I interferon production in general, and CXCL10 production specifically, as a prerequisite for hematopoietic cell function and adult mouse survival in a low-lethality polymicrobial sepsis model [6,7]. In most of these latter studies, the lethality of the sepsis models was considerably less than used by Herzig and colleagues. The disparity between Herzig and colleagues' findings [1] and those of prior reports [3-7] may well be dependent upon the magnitude of the early inflammatory response, and should raise concern that CXCR3/CXCL10 signaling is more complex than previously suggested, and undoubtedly plays both beneficial and adverse roles in outcome to sepsis.

When Herzig and colleagues' results are juxtaposed against the backdrop of known CXCR3/CXCL10 functions, and more importantly the historical landscape of failed clinical trials with inhibitors of inflammation, several questions become paramount. What are the theoretical advantages of CXCR3/CXCL10 as a therapeutic target compared with other inflammatory targets? Importantly, does severe murine peritoneal sepsis recapitulate human sepsis sufficiently to reasonably expect similar results in humans with severe sepsis and septic shock? And most importantly, what can we learn about the mechanism of protective immunity by CXCR3/ CXCL10 action?

Given the ever growing complexity and reticular nature of human sepsis, is it wise to continue to pursue single therapeutic interventions for the multidimensional sepsis syndrome $[8,9]$ ? In a 21 st century landscape stained by the history of failed therapeutic interdiction [10], the authors should be applauded for their demonstration that CXCR3 blockade begun 6 hours after the onset of sepsis is beneficial to survival. The fact that CXCR3 inhibition after the onset of sepsis improves survival gives the potential therapy a practical and broad appeal. Herzig and colleagues' findings clearly show the detrimental side 
of exaggerated CXCR3 signaling in severe sepsis with high mortalities. In the clinical setting, there is little disagreement that what we call 'severe sepsis' is presently so vaguely defined that our study populations are too heterogenous to optimize therapeutic efficacy. Although the authors demonstrate a $40 \%$ improvement in survival, the harsh fact remains that existing individual animal models are rather poor surrogates of human sepsis $[8,10-$ 12]. Even though the cecal ligation and puncture model is generally accepted to best replicate human peritoneal sepsis (the 'gold standard' to many), several clinical intangibles, such as pre-existing comorbidities, age, continuous fluid resuscitation, nutritional support, antibiotic therapy, and operative intervention, make human sepsis more complex, and routinely difficult to replicate in mice [13]. Juxtaposed with the murine versus human sepsis conundrum stands the mortality disparity between the authors' cecal ligation and puncture model, which was $90 \%$ across the board and 50\% in the group that showed a benefit, compared to an overall mortality of approximately $25 \%$ in human sepsis $[8,14,15]$.

Compared to other, older anti-inflammatory therapeutic targets, CXCR3 is a relative new-comer with only a handful of studies dealing with its role in polymicrobial sepsis. More detailed investigations are warranted to better understand the receptor's scope of action and full therapeutic potential. In their prior report, Herzig and colleagues partially addressed this issue by implicating CXCR3 blockade in reducing peritoneal lymphocyte recruitment and interleukin- 6 and macrophage inflammatory protein 2 production [2]. However, given the multitude of cellular responses and inflammatory mediators that orchestrate the sepsis syndrome, more detailed investigations are required before we will truly understand the mechanism of CXCR3 blockade and its therapeutic potential. However, Herzig and colleagues are to be congratulated on advancing the field, and drawing attention to the important role that individual chemokines play in sepsis survival.

\section{Abbreviations}

CXCR3, CXC chemokine receptor 3 .

\section{Competing interests}

The authors declare that they have no competing interests.

\section{Acknowledgements}

MJD was supported by a training grant in burn and trauma research (T32 GM08431) awarded by the National Institute of General Medical Sciences, USPHS.

Published: 21 November 2012

References

1. Herzig DS, Guo Y, Fang G, Toliver-Kinsky TE, Sherwood ER: Therapeutic efficacy of CXCR3 blockade in an experimental model of severe sepsis. Crit Care 2012, 16:R168.

2. Herzig DS, Driver BR, Fang G, Toliver-Kinsky TE, Shute EN, Sherwood ER: Regulation of lymphocyte trafficking by CXC chemokine receptor 3 during septic shock. Am J Respir Crit Care Med 2012, 185:291-300.

3. Ness TL, Hogaboam CM, Strieter RM, Kunkel SL: Immunomodulatory role of CXCR2 during experimental septic peritonitis. I Immunol 2003, 171:3775-3784.

4. Zeng X, Moore TA, Newstead MW, Deng JC, Kunkel SL, Luster AD, Standiford TJ: Interferon-inducible protein 10, but not monokine induced by gamma interferon, promotes protective type 1 immunity in murine Klebsiella pneumoniae pneumonia. Infect Immun 2005, 73:8226-8236.

5. Cuenca AG, Wynn JL, Kelly-Scumpia KM, Scumpia PO, Vila L, Delano MJ, Mathews CE, Wallet SM, Reeves WH, Behrns KE, Nacionales DC, Efron PA Kunkel SL, Moldawer LL: Critical role for CXC ligand 10/CXC receptor 3 signaling in the murine neonatal response to sepsis. Infect Immun 2011, 79:2746-2754

6. Kelly-Scumpia KM, Scumpia PO, Delano MJ, Weinstein JS, Cuenca AG, Wynn $J L$, Moldawer LL: Type I interferon signaling in hematopoietic cells is required for survival in mouse polymicrobial sepsis by regulating CXCL10. J Exp Med 2010, 207:319-326.

7. Kelly-Scumpia KM, Scumpia PO, Weinstein JS, Delano MJ, Cuenca AG, Nacionales DC, Wynn JL, Lee PY, Kumagai Y, Efron PA, Akira S, Wasserfall C, Atkinson MA, Moldawer $L L$ : $B$ cells enhance early innate immune responses during bacterial sepsis. J Exp Med 2011, 208:1673-1682.

8. Hotchkiss RS, Karl IE: The pathophysiology and treatment of sepsis. N Eng/J Med 2003, 348:138-150.

9. Hotchkiss RS, Opal S: Immunotherapy for sepsis - a new approach against an ancient foe. N Engl J Med 2010, 363:87-89.

10. Deans KJ, Haley M, Natanson C, Eichacker PQ, Minneci PC: Novel therapies for sepsis: a review. J Trauma 2005, 58:867-874.

11. Natanson C, Esposito CJ, Banks SM: The sirens' songs of confirmatory sepsis trials: selection bias and sampling error. Crit Care Med 1998, 26:1927-1931.

12. Natanson C, Hoffman WD, Suffredini AF, Eichacker PQ, Danner RL: Selected treatment strategies for septic shock based on proposed mechanisms of pathogenesis. Ann Intern Med 1994, 120:771-783.

13. Deitch EA: Animal models of sepsis and shock: a review and lessons learned. Shock 1998, 9:1-11.

14. Martin GS, Mannino DM, Eaton S, Moss M: The epidemiology of sepsis in the United States from 1979 through 2000. N Engl J Med 2003, 348:1546-1554.

15. Angus DC, Linde-Zwirble WT, Lidicker J, Clermont G, Carcillo J, Pinsky MR: Epidemiology of severe sepsis in the United States: analysis of incidence, outcome, and associated costs of care. Crit Care Med 2001, 29:1303-1310.

doi:10.1186/cc11818

Cite this article as: Delano MJ, Moldawer LL: CXCR3 blockade: a novel antisepsis approach? Critical Care 2012, 16:176. 